\title{
TRATAMIENTO RESTAURADOR DENTAL EN PACIENTE CONSUMIDOR DE CANNABIS, REPORTE DE UN CASO.
}

A case report of dental restorative treatment in cannabis user.

\author{
María José Massón-Palacios ${ }^{1}$, Alessandro Dourado Loguércio ${ }^{2}$, Ana Armas Vega ${ }^{3}$. \\ ${ }^{1}$ Investigadora Centro de Investigación de Salud Pública y Epidemiología Clínica (CISPEC). Universidad UTE. \\ Quito-Ecuador \\ ${ }^{2}$ Profesor Departmento de Odontología Resturadora, Universidad Estadual de Ponta Grossa, Paraná, Brazil, Docente invitado \\ Universidad UTE. Quito- Ecuador. \\ ${ }^{2}$ Universidad UTE. Quito-Ecuador
}

\section{Resumen}

El consumo de Cannabis está asociado con el incremento en la presencia de placa bacteriana, desencadenando en el aumento de riesgo cariogénico y enfermedad periodontal, los pacientes que refieren este hábito se consideran de difícil manejo. El objetivo de este artículo es describir la intervención multidisciplinaria que recibió un paciente que refiere como hábito consumo frecuente de cannabis. Se realizó una rehabilitación oral integral a un paciente de 25 años de edad, sexo masculino, quien presenta múltiples lesiones cariosas, necrosis pulpar y varias fracturas dentales, por accidente en bicicleta. El tratamiento incluyó procedimientos de raspado y alisado radicular, eliminación de focos infecciosos y la ejecución de procedimientos endodónticos, se realizó la preparación dental biomecánica para recibir coronas con cofia de zirconio y recubrimiento de porcelana sobre pernos colados metálicos en los incisivos laterales, carillas de resina compuesta en los incisivos centrales. Los procedimientos ejecutados permitieron mejorar la calidad de vida del paciente.

Palabras clave: Cannabis, Trastornos Relacionados con Sustancias, Rehabilitación oral.

\begin{abstract}
The clinical case report from this article is about a complete oral rehabilitation of a 25 years old male patient, who had a bicycle accident thirty days before visiting the dental clinic. The patient presented dental fractures, pulp necrosis and multiple carious lesions; additionally, the patient used cannabis. The team proposed a multidisciplinary treatment, which included adjustment of the oral environment by performing conventional root scaling and planning, eliminating septic focuses and performing endodontic procedures in the affected teeth. In addition, metal screws with zirconium crowns were placed in the lateral incisors and composite veneers in the central incisors. All the procedures performed in this patient were successful, however, the total success of this treatment demands the patient's commitment, as there is a high risk of developing caries.
\end{abstract}

Key words: Cannabis, Substance-Related Disorders, Oral Rehabilitation.

\section{Introducción}

El cannabis conocido como Marihuana, constituye una droga recreativa de uso común en varios países, con alrededor de 192 millones de consumidores a nivel mundial, ${ }^{1}$ su consumo está asociado con el incremento en la presencia de placa bacteriana desencadenando en caries y enfermedad periodontal, debido a la falta de higiene oral diaria, mayor consumo de bebidas cariogénicas, deficiencia nutricional y ausencia de controles odontológicos. ${ }^{2}$

Estudios han demostrado que el $69,6 \%$ de los usuarios de cannabis experimentaron xerostomía casi inmediatamente luego del consumo, además está documentada una mayor incidencia de leucoedema, ${ }^{2,3}$ incremento en la posibilidad de presentar cáncer oral, por la presencia de fenoles, cloruro de vinilo e hidrocarburos aromáticos en el cannabis. En comparación con el cigarrillo de tabaco filtrado se observó un depósito aproximado de 4 veces más alquitrán en el tracto respiratorio,${ }^{3}$ lo que desencadena lesiones displásicas del tipo leucoplasia y eritroplaquia. ${ }^{4}$ A nivel periodontal, la presencia de gingivitis severa, enfermedad periodontal destructiva, y agrandamiento gingival se encuentran íntimamente relacionados con el consumo del cannabis. ${ }^{5,6}$ 
Es imprescindible la confirmación de uso de la droga previo a cualquier intervención ${ }^{7}$ debido a que existen interacciones farmacológicas con compuestos tales como la adrenalina presente en el anestésico local pueden desencadenar, produciendo reacciones adversas como la taquicardia, ${ }^{2}$ condición que se agrava por el estado emocional del paciente, que cumple un rol fundamental en el proceso del tratamiento ${ }^{8}$ más aún en aquellos pacientes con hábitos perniciosos. ${ }^{9}$ Independientemente de la situación clínica observada la correcta planificación de los procedimientos resulta crucial para la adherencia al tratamiento por parte del paciente, para cumplir con los objetivos de devolver la estética y función. ${ }^{10}$

Frente a lo expuesto, el objetivo de este articulo es reportar el caso clínico con manejo interdisciplinario en un paciente consumidor de cannabis, quien presenta múltiples lesiones cariosas, necrosis pulpar y varias fracturas dentales.

\section{Reporte de caso}

Paciente masculino de 25 años, acude a consulta de rehabilitación oral, por fracturas dentarias en el sector de los incisivos superiores, refiere traumatismo facial en zona antero superior, producida por impacto con el manubrio de una bicicleta durante un accidente, sucedido un mes atrás. Paciente refiere consumir marihuana al menos cinco veces por semana, ingesta de dieta altamente cariogénica, con 8 a 12 momentos de azúcar diarios, prácticas de higiene bucal deficientes; sin antecedentes de enfermedades sistémicas diagnosticadas o antecedentes patológicos familiares.

El examen clínico intraoral realizado muestra agrandamiento gingival papilar y marginal generalizado inducidas por la presencia de abundante placa dental, a nivel de ambos maxilares, la gingiva y mucosa palatina se presenta de aspecto nodular; fracturas horizontales a nivel cervical y necrosis pulpar de dientes 12 y 22 ; los dientes 11 y 21 , presentaron fracturas con forma semilunar abarcando tercio incisal y medio de la corona sin lesión pulpar; se observó la presencia de una barra metálica a manera de férula en el maxilar y tres pierciengs intraorales localizados en la lengua y frenillo lingual. (figura 1 y 2).

Los hallazgos clínicos y radiográficos fueron discutidos con el paciente, se explicó plan de tratamiento a ejecutarse, el cual fue aprobado tras la firma de consentimiento informado. Se procedió a la remoción de la férula metálica y adecuación del medio mediante profilaxis dental, se realizó instrucción de hábitos de higiene bucal con énfasis en la técnica de cepillado, el examen periodontal confirmó el diagnóstico de gingivitis inducida por placa dental con agrandamiento gingival influenciado por fármacos, no se observó movilidad

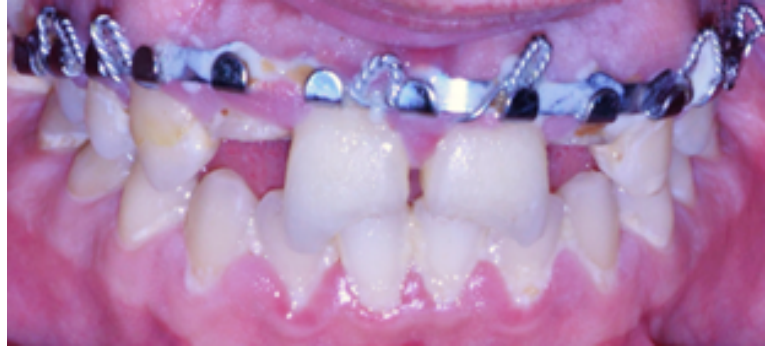

Fig. 1. Situación clínica del paciente en la primera cita Fuente: Registro fotográfico del autor.

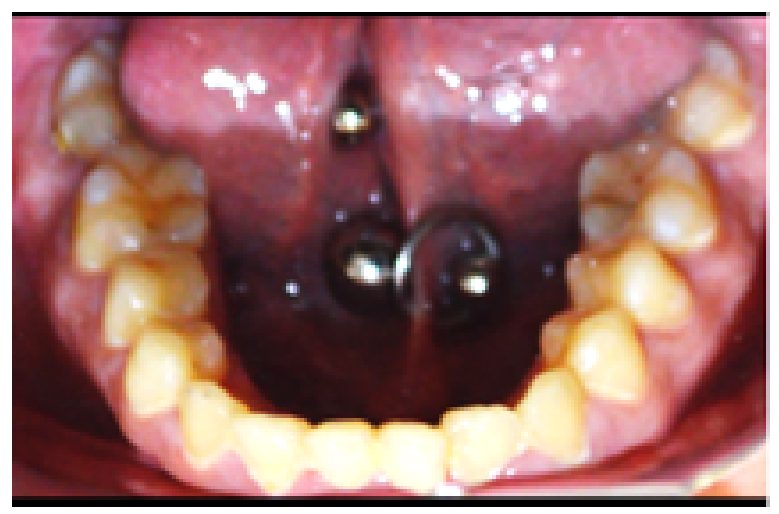

Fig. 2. Situación clínica del paciente en la primera cita Fuente: Registro fotográfico del autor.

dentaria.

Posteriormente, se ejecutó alargamiento de corona clínica de dientes 12 y 22, luego de 10 días de la intervención periodontal, se evidenció una mejora considerable en el estado gingival, se realizó interconsulta con endondoncia para ejecución de tratamiento endodóntico en los dientes 12 y 22. Concluido el procedimiento endodóntico, se procedió a la toma de fotografías intra-orales, las cuales fueron analizadas mediante la herramienta informática Digital Smile Design (DSD) (figura 3), se determinó los procedimientos restauradores estéticos a seguir, se confeccionó encerado diagnóstico sobre modelos obtenidos previamente de los maxilares del paciente, mismos que fueron fijados en articulador semiajustable.

Se realizó mock-up con resina compuesta sobre los dientes incisivos superiores, previo a discutir las preferencias del paciente en cuanto a la forma, tamaño y materiales a ser empleados. A partir del mock-up confeccionado fue obtenida una llave de silicona que permitió la elaboración de carillas directas en resina compuesta previa preparación de la superficie coronaria remanente, colocación de hilo retractor y aislamiento absoluto, las diferentes capas de resina (AMARIS, VOCO, tonos 03, TN, TA) se colocaron 


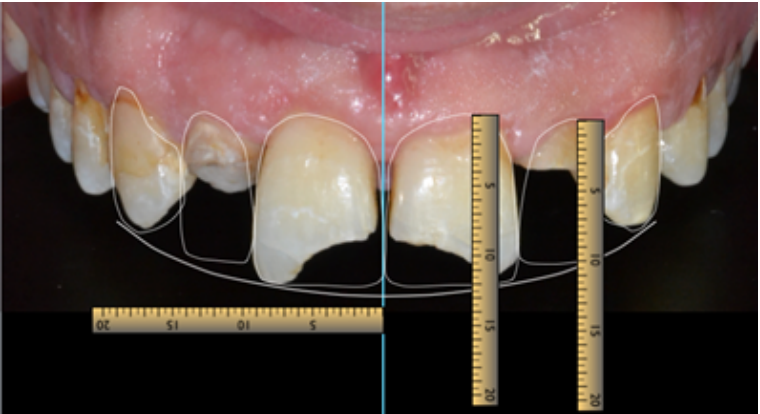

Fig. 3. Diseño de sonrisa con DSD (herramienta Digital Smile Design) Fuente: Registro fotográfico del autor.

respetando tonalidades existentes. (figura 4)

Para la confección de los pernos colados en las raíces de los dientes 12 y 22, se realizó la desobturación de los conductos radiculares y el registro intraconducto mediante Duralay, este se envió a fundición en metal, una vez recibidos los pernos colados fueron cementados bajo aislamiento absoluto, y se realizó el retallado de los muñones y el registro mediante materiales elastómericos de alta precisión para la confección de coronas en los dientes 12 y 22, colocándose posterior a la impresión coronas provisionales con materiales poliméricos del tipo bisacril sobre los dientes preparados.

Considerando que las coronas en los dientes 12 y 22 serían elaboradas con cofia de zirconio y recubrimiento de porcelana, pruebas de adaptabilidad de los diferentes elementos fueron realizados en cada una de las sesiones, además se realizaron las restauraciones con resina (Filtek Z350®, 3M ESPE) en las lesiones cariosas existentes, en cada cita se reforzó las técnicas de higiene bucal, asesoramiento y motivación nutricional. Concluida la confección de las coronas, se verificó su asentamiento en la superficie dental y compatibilidad con los tejidos circundantes, se realizó la cementación de los elementos protésicos siguiendo procedimientos y protocolos establecidos. Se evidenció armonía en la estética dental, mejoría en la salud gingival y disminución en la frecuencia de hábitos perniciosos. (figura 5)

\section{Discusión}

$\mathrm{Si}$ bien no es factible afirmar que el tratamiento restaurador estético ejecutado en el paciente fue el responsable por la disminución en el consumo del cannabis referido, fueron evidentes las mejoras en cuanto a su salud bucal y estado anímico que los procedimientos ejecutados desencadenaron. Un paciente adicto a cualquier droga presenta cierto abandono a sus hábitos de higiene y descuido en su alimentación ${ }^{11}$ como fue comprobado, la existencia de un traumatismo y la determinación del paciente permitieron obtener un resultado positivo en cuanto a su salud bucal y a los tratamientos ejecutados.

Son varios los factores determinantes para que un individuo llegue a la dependencia de una droga, los psicosociales constituyen los determinantes y motivadores de estas adicciones, ${ }^{3}$ sin embargo circunstancias graves como accidentes como el referido en el caso relatado pueden desencadenar cambios en el paciente que a la larga resultan importantes, ${ }^{12}$ la apertura recibida por parte del paciente al aceptar las sugerencias y motivaciones sobre higiene oral y hábitos alimenticios son importantes en este tipo de pacientes y pueden constituir factor determinante del éxito reportado.

Las acciones protésicas y quirúrgicas ejecutadas si bien no son diferentes a las que en cualquier paciente pueden realizarse, el conocer las interferencias que determinadas drogas producen con los fármacos empleados en odontología es básico, ${ }^{13}$ los procedimientos relatados fueron ejecutados por especialistas y fueron necesarios para lograr un tratamiento integral.

La elección de conjugar procedimientos directos con indirectos es bien aceptada en la literatura y es dependiente de la cantidad de tejido y de la salud pulpar del paciente, la selección de coronas estéticas de zirconio y cerámica constituyen elementos fundamentales cuando de conservación a los tejidos circundantes se trata, en especial cuando estos han sufrido algún tipo de intervención periodontal $^{14}$ como el alargamiento dental realizado en el paciente. Este tipo de casos requieren un asesoramiento continuo si pensamos mantener a largo plazo el éxito de los tratamientos ejecutados.

Los efectos agudos del consumo de cannabis sobre el estado de conciencia llevan al individuo a una leve euforia y relajación, alteraciones de la percepción, con efectos nocivos crónicos sobre el sistema respiratorio que desencadenan tos crónica, producción de esputo y sibilancias; mayor frecuencia de bronquitis aguda, obstrucción crónica del flujo de aire. ${ }^{12}$ A nivel del sistema cardiovascular, el consumo del cannabis aumenta la frecuencia cardíaca y la presión arterial supina; aumenta el gasto cardíaco y disminuye la resistencia vascular periférica, desencadenando disminución de la presión arterial supina, el aumento del volumen sanguíneo y la disminución de la frecuencia cardíaca. ${ }^{15} \mathrm{La}$ asociación entre el consumo crónico de cannabis y el agrandamiento gingival es un padecimiento frecuente en este tipo de pacientes $^{5}$ en el caso que aquí se presentan el agrandamiento gingival fue principalmente papilar y marginal ${ }^{16}$ localizado principalmente en los dientes anteriores se suma al hecho de presentar dentro de boca tres piercings los cuales constituyen 


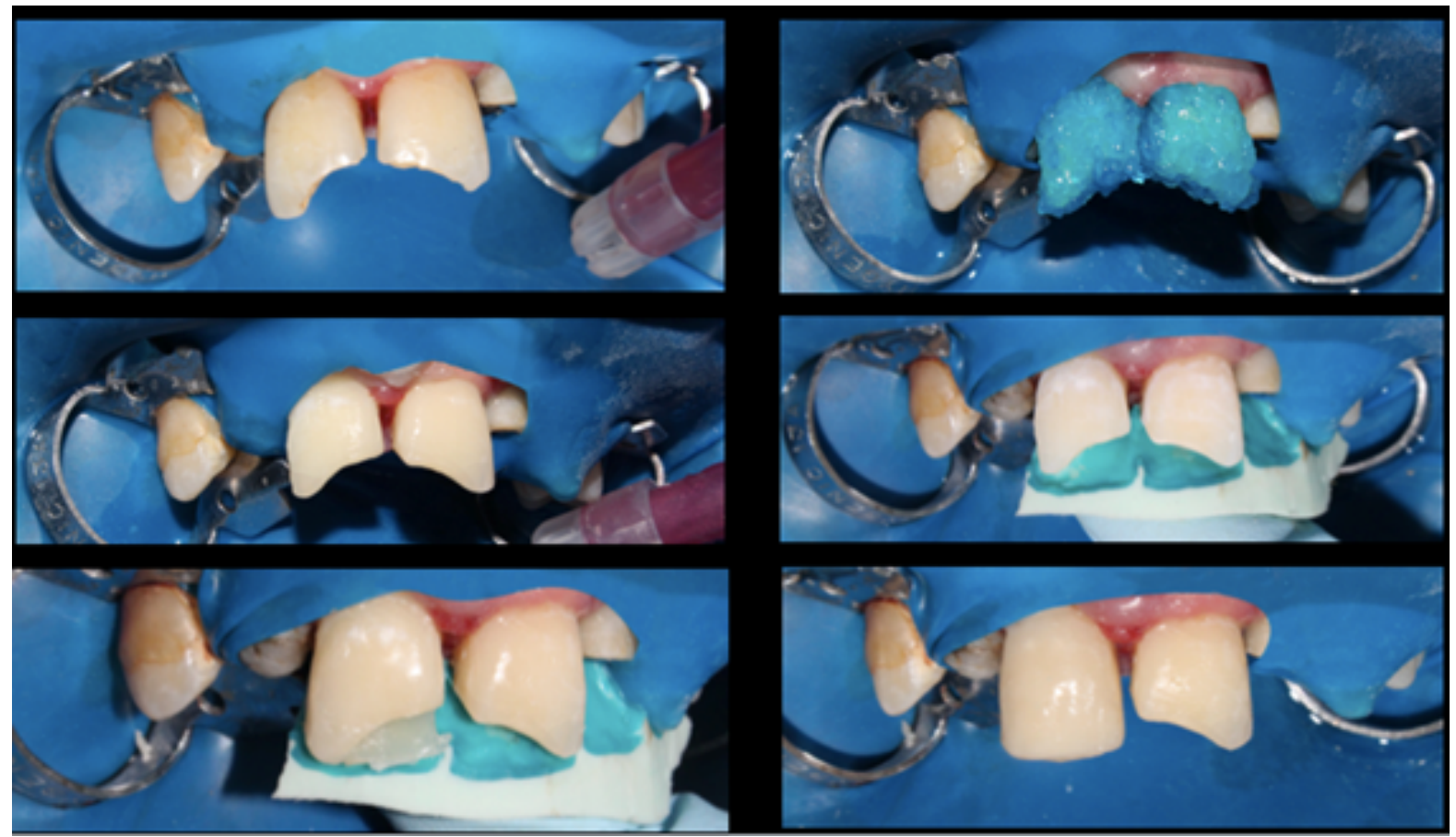

Fig. 4. Diseño de sonrisa con DSD (herramienta Digital Smile Design) Fuente: Registro fotográfico del autor.

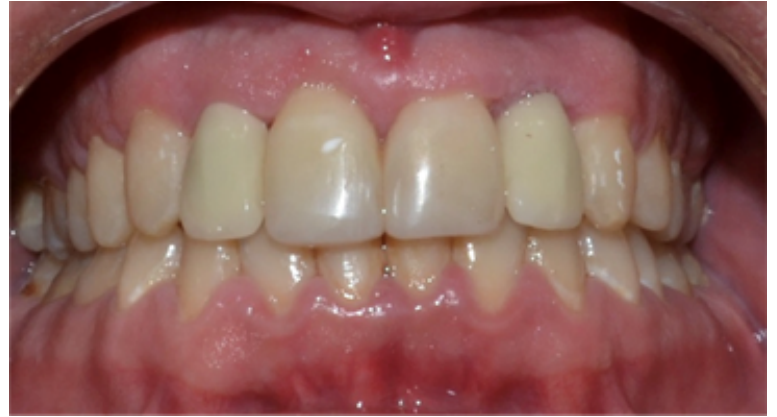

Fig. 5. Estado clínco del paciente en el momento de la terminación del tratamiento planificado. Fuente: Registro fotográfico del autor.

elementos de acumulo de placa y por tanto de bacterias ${ }^{17}$

A pesar de que el cannabis es considerado una droga "liviana" no más perjudicial que el café o el tabaco, su consumo relacionado al incremento de la temperatura del humo de combustión puede ser causa de quemaduras ${ }^{18}$ desencadenantes de una estomatitis nicotínica y uvulitis. ${ }^{19} \mathrm{La}$ educación al paciente por parte del profesional odontólogo es básica cuando se trata de prevenir y controlar el consumo de fármacos y sustancias no controladas en su uso.

La importancia de un manejo integral del paciente que incluya regulación de hábitos, manejo de riesgo de caries y rehabilitación oral, supone un reto para los odontólogos clínicos por la poca colaboración de este tipo de pacientes y su negatividad frente a eliminar hábitos destructivos como el consumo de sustancias ilícitas.

Conflicto de intereses: Los autores del presente estudio manifiestan que no existe ningún conflicto de intereses en relación al tema de estudio.

\section{Referencias}

1 Oficina de las Naciones Unidas contra la Droga y el Delito - UNODC. Informe Mundial Sobre las Drogas 2018. Resumen, conclusiones y consecuencias en materia de políticas. UNODC Res. 2018;24.

2 Teoh L, Moses G, McCullough M. Oral manifestations of illicit drug use. Aust Dent J. 2019;0-2.

3 Cho CM, Hirsch R, Johnstone S. General and oral health implications of cannabis use. Aust Dent J. 2005;50(2):70-4.

4 Zhang LR, Morgenstern H, Greenland S, Chang S-C, Lazarus $\mathrm{P}$, Teare MD, et al. Cannabis smoking and lung cancer risk: Pooled analysis in the International Lung Cancer Consortium. Int J cancer. 2015 Feb;136(4):894-903.

5 Chisini LA, Cademartori MG, Francia A, Mederos M, Grazioli G, Conde MCM, et al. Is the use of Cannabis associated with periodontitis? A systematic review and meta-analysis. J Periodontal Res. 2019;54(4):311-7. 
6 López R, Baelum V. Cannabis use and destructive periodontal diseases among adolescents. J Clin Periodontol. 2009;36(3):185-9.

7 Grafton SE, Huang PN, Vieira AR. Dental treatment planning considerations for patients using cannabis A case report. J Am Dent Assoc [Internet]. 2016;147(5):354-61. Available from: http://dx.doi.org/10.1016/j.adaj.2015.11.019

8 Arrieta Vergara K, Díaz Cárdenas S, Verbel Bohórquez J, Hawasly Pastrana N. Factores asociados a sintomatología clínica de miedo y ansiedad en pacientes atendidos en Odontología. Rev Clínica Med Fam. 2013;6(1):17-24.

9 Asián-Nomberto1 DJ. Implicancias odontológicas en el uso de drogas en adolescentes. Rev Odontol Pediátrica. 2018;10(2):122-30.

10 Lamas-Lara C, Alvarado-Menacho S, Angulo de la Vega G. Rehabilitación estética del sector anterior con cerámica libre de metal. Reporte de caso. Rev Estomatológica Hered. 2014;23(4):210.

11 Pablo C, Marcela I, Lila S. Salud bucal y hábitos de consumo de sustancias psicoactivas en adolescentes y jóvenes drogo dependientes en recuperación. Odontoestomatologia. 2012;14(20):49-59.

12 Leggett T. Examen de la situación mundial del cannabis [Internet]. Vol. 58, Boletín de estupefacientes. 2006. 1-169.

13 Shekarchizadeh H, Khami MR, Mohebbi SZ, Ekhtiari H, Virtanen JI. Oral health of drug abusers: A review of health effects and care. Iran J Public Health. 2013;42(9):929-40.

14 Romero González C, Hidalgo García C, Hidalgo Hidalgo S, Hidalgo Pacheco A. Rehabilitación de dientes con fracturas infragingivales. reporte de un caso. Arch Médico Camagüey. 2006;10(6).

15 María D, Cristar F. ¿Qué deberíamos saber sobre los efectos cardiovasculares de la inhalación de marihuana? Rev Uruguaya Cardiol. 2014;29(1):32-6.

16 Baddour HM, Audemorte TB, Layman FD. The occurrence of diffuse gingival hyperplasia in a patient using marijuana. J Tenn Dent Assoc. 1984 Apr;64(2):39-43.

17 Jach Ravelo M, García Báez F, Hernández Núñez A, Vázquez Fiallo C. Los piercing orales y sus complicaciones en estomatología. Medimay [revista en Internet]. 2017;23(2):219-30.

18 Mederos M, Francia A, Chisini LA, Grazioli G, Andrade E. ACTUALIZACIÓN Influencia del consumo de cannabis en la enfermedad periodontal: una Scoping Review. Odontoestomatologia. 2018;20(31):4-15.

19 Rawal SY, Dabbous MK, Tipton DA. Effect of cannabidiol on human gingival fibroblast extracellular matrix metabolism: MMP production and activity, and production of fibronectin and transforming growth factor beta. J Periodontal Res. 2012 Jun;47(3):320-9. 
